\title{
Association of Topical Capsaicin Exposure Dosage and Its Influence on Macrophages and Neutrophils in Periodontal Tissue
}

\author{
Ratna Mustriana ${ }^{1}$, Haryono Utomo ${ }^{2}$ and Purwo Sri Rejeki ${ }^{3}$ \\ ${ }^{l}$ Basic Medical Science Student, Faculty of Medicine Universitas Airlangga, Surabaya, Indonesia \\ ${ }^{2}$ Department of Orthodontic Dentistry, Faculty of Dentistry Universitas Airlangga, Surabaya, Indonesia \\ ${ }^{3}$ Department of Physiology, Faculty of Medicine Universitas Airlangga, Surabaya, Indonesia \\ \{purwo-s-r,apji\}@fk.unair.ac.id
}

Keywords: Capsaicin, Gingivomucosal, Periodontal Tissue.

\begin{abstract}
In the community, a lot of enthusiasts like spicy food consumption. Spicy flavors in foods containing capsaicin cause heat stimulation in the oral cavity, especially periodontal tissue. Capsaicin in rat gingivomucosal tissue (Rattus novergicus) can induce an inflammatory reaction. The purpose of this study was to prove that capsaicin exposure can affect the occurrence of inflammation in periodontal tissue (periodontitis). Thirty-three Rattus males aged 8-12 weeks and weighing between 200-250 gram were divided into three groups, namely control group K, group treatment 1 (P1) with a $10 \mathrm{mg}$ dose of capsaicin gel, and group treatment 2 (P2) with a $20 \mathrm{mg}$ dose of capsaicin gel. The capsaicin gel was applied to the mandibular anterior mucosa, then was subjected to glass ionomer cement (GIC) and left for 14 days. After treatment, the anterior mandible labial mucosa was taken and HE was performed, then the data were analyzed using Kruskal Wallis. The results of histopathological examination (HPA) showed changes in the number of macrophages and neutrophils. In group $\mathrm{K}$, the number of macrophages was $(11.36 \pm 1.433)$ and the number of neutrophils was $(10.91 \pm 0.701)$; in group P1 the number of macrophages was $(13.27 \pm$ $1.679)$ and the number of neutrophils was $(8.45 \pm 0.802)$; and in group $\mathrm{P} 2$ the number of macrophages was $(16.18 \pm 2.136)$ and the number of neutrophils was $(6.18 \pm 0.751)$. At day 14 the inflammation in the periodontal tissue was apparent with macrophage and neutrophil enlargement compared to the control group $(\mathrm{p}<0.05)$. These results prove that there was an effect of capsaicin exposure of chronic inflammation in periodontal (white) rat periodontal tissue (Rattus novergicus).
\end{abstract}

\section{INTRODUCTION}

Capsaicin (8-methyl-N-vanilyl-6-nonenamide) is included in capsaicinoids, spicy flavoring chemicals present in plants, such as chili (Hayman and Thurs, 2008). Spicy flavors in foods containing capsaicin can cause heat stimulation in the oral cavity (Sharma et al., 2013). Hot stimulation of the oral cavity may increase the sensitivity of dentin (Bode and Dong, 2011). This dentine sensitivity can affect the temperature in the oral cavity, which if not promptly treated that can damage the periodontal tissues (Anand and Bley, 2011). Initial symptoms that occur in periodontal tissue are increased sensitivity dentin or hypersensitivity dentin. Dentin hypersensitivity is triggered by tactile stimuli, vapors, chemicals, and hot or cold stimuli (Cunha-Cruz et al., 2013). Dentine hypersensitivity which is not treated immediately can damage periodontal tissue and cause systemic health problems, such as coronary heart disease, stroke, diabetes, and respiratory diseases. Besides the interrelationships between periodontal infection and host immune response, environmental, physical, and psychosocial stress factors can also affect periodontal tissue and alter the appearance of the disease. In general, abnormalities in some of the above do not trigger chronic periodontitis, but may exacerbate, accelerate, or even increase progression until it ultimate destruction of periodontal tissue. As the nervous system contributes to the pathophysiology of various diseases, it is now evident that neuropeptides may also be involved in the initiation and progression of oral diseases. Periodontitis, one of the major inflammatory oral diseases, is characterized by inflammation of the gingiva and destruction of alveolar bone (Lebukan, 2013). The clinical prevalence of dentin hypersensitivity is $4 \%$ 
to $74 \%$ (Ye et al., 2012). The clinical prevalence of dentin hypersensitivity is $4 \%$ to $74 \%$ (Cunha-Cruz et al., 2013), and a prevalence of about $15 \%$ indicates dentine sensitivity, while $75 \%$ indicates dentine sensitivity due to exposure to temperature (Pashley, 2013).

Capsaicin belonging to the vanilloid family is bound to a receptor called vanilloid receptor subtype 1 (VR 1) stimulated by heat and physical abrasion that can enter the cell membrane and enter the cell when activated (Avellan et al., 2006). The receptor on the capsaicin is a Transient Receptor Potential Vanilloid subtype 1 (TRPV1) (Mishra and Hoon, 2010). TRPV1 is a member of the TRPV subfamily that acts as a non-selective cation graft activated by heat, proton, and harmless vanilloids such as capsaicin. TRPV1 is widely expressed in peripheral sensory nerves and acts as a nociceptor (i.e., pain receptors)(Sharma et al., 2013).

The heat stimulation caused by capsaicin makes the temperature of the oral cavity rise. This causes heat/pain excitement by nociceptors or thoracepeptors that can stimulate the salivary glands (Haggard and de Boer, 2014). The emergence of these stimuli is caused by the parasympathetic nerves. Then, this parasympathetic nerve stimulates vasodilation caused by the release of VIP (vasoactive Intestine polypeptide). This vasodilation is also caused by resistance from the sympathetic center of the posterior hypothalamus causing vasoconstriction resulting in vasodilation. As the process progresses, it will automatically lead to increased saliva flow (Austah et al., 2016).

The stimulus of capsaicin is found to irritate and increase sensitivity in the skin, as capsaicin has been shown to be a noninvasive method for activating nociceptive $\mathrm{C}$ type fibers on human skin. This reaction is allegedly caused by the release of substance-induced capsaicin from the peripheral sensory nerve fibers of type $\mathrm{C}$ which may cause pain and a burning sensation (Sharma et al., 2013).

Within the oral cavity, capsaicin stimulation may increase the release of substance $\mathrm{P}$ and calcitonin gene-related peptide (CGRP) (Austah et al., 2016).

The release of this peptide from the peripheral terminal of the sensory nerves followed by increased microvascular permeability may lead to neurogenic inflammation (Anand and Bley, 2011). In addition, capsaicin stimulation of the alveolar mucosa may increase gingival crevicular fluid (GCF) in order to activate matrix metalloprotein 8 (MMP-8) and chronic inflammation of gingival tissue characterized by neutrophil and macrophage cells
(Sharma et al., 2013).<smiles>COc1cc(CNC(=O)CCCC/C=C/C(C)C)ccc1O</smiles>

Figure 1: Chemical Structure of Capsaicin.

In the community, it was found that consumption of spicy foods increases the sensitivity of dentine to temperature in periodontal tissues. The 2008 Avellan study states that capsaicin injection in rat gingivomucosal tissue (Rattus novergicus) may induce an inflammatory reaction (Avellan, 2008). This study aimed to prove the effects of topical capsaicin exposure associated with doses on periodontal tissues that are still unknown. Capsaicin gel was applied once for two weeks on periodontal tissue which is not its nature physiologic because this study was to prove the adjuvant mechanism of periodontal disease. Capsaicin gel was applied on rat periodontal tissue by filling with glass ionomer cement (GIC) which causes capsaicin to remain in the periodontal tissue. These applications allow periodontal tissues to experience chronic inflammation.

\section{METHODS}

The type of research used was a true experiment with a posttest-only control group design. In this design model, the experimental group and the control group were formed by a random procedure, so that both could be considered equivalent. Subsequently, the experimental group was given treatment. After the treatment was given within a certain time period, measurement of the dependent variable in both groups was performed, and the result was compared (Sugiyono, 2012).

In this study, the experimental unit used was in the form of the white rat with 33 male adult rats (Rattus novergicus) from the Department of Biochemistry of Medicine, Faculty of Medicine, Universitas Airlangga. The age of the rats was 8-12 weeks and weighed between $200-250 \mathrm{~g}$. Prior to the research the rats were acclimatized for 1 week and placed in a comfortable environment, which was not rowdy at a room temperature of $27^{\circ} \mathrm{C}$.

The study was conducted in accordance with the Ethical Committee No. 055 / HRECC.FODM / V / 2017 Universitas Airlangga Faculty of Dental Medicine Health Research Ethical Clearance Commission. The rats were divided into three 
groups: negative control group (mouse + vaseline), treatment group 1 (rat + capsaicin gel $10 \mathrm{mg} /$ minimum dose), and treatment group 2 (rat + capsaicin gel $20 \mathrm{mg} /$ maximum dose). The dose of capsaicin given to the mice was in accordance with the calculations by the Department of Pharmacology of Universitas Airlangga.

Capsaicin (Capsaicin - $\geq 95 \%$, from Capsicum sp, Sigma Aldrich) gel was made by mixing capsaicin (powder, $50 \mathrm{mg}$ ) with $950 \mathrm{mg}$ of polyethylene glycol (PEG) gel. The minimum dose of capsaicin applied was $10 \mathrm{mg}$ to the mandibular anterior mucosa of the rat, and the maximum dose applied was $20 \mathrm{mg}$ in the mandibular anterior mucosa. Prior to the application of capsaicin, the mice were anesthetized with ketamine + xylazine with a combination of ketamine + xylazine 1: 1 and a ketamine dose of $50 \mathrm{mg} / \mathrm{kgBB}+$ xylazine 10 $\mathrm{mg} / \mathrm{kgBW}$.

The treatment procedure in each group was performed once and then closed with patch material. In the control group vaseline + filling glass ionomer cement (GIC) was applied, in treatment group 1 capsaicin gel $(10 \mathrm{mg})+$ GIS filling was applied, and in treatment group 2 capsaicin gel $(20 \mathrm{mg})+$ filling GIC was applied. After 14 days of exposure, the mice were sacrificed to obtain the lower anterior mucosal tissue and fixed in $10 \%$ formalin, then sent to the Anatomical Pathology Laboratory for processing and analysis.

If the data variance was different (not homogeneous, $\mathrm{p}<0.05)$ then the difference test using Kruskal Wallis test was used. Data analysis was performed using the non-parametric test Kruskal Willis. In this study, data analysis aimed to determine the ratio of the number of macrophages and neutrophils in each group.

\section{RESULTS}

Anatomical histopathology examination (HPA) obtained the number of neutrophils and macrophages as shown in Table 1 as follows. Description of the research data after treatment can be seen in Table 1. This result was obtained from measurement of the number of macrophages based on the Kruskal-Willis test with $\mathrm{p}=0.001$. The results of the above data found significant results, meaning there was a difference in the number of macrophages in each group between the control group, treatment group 1, and treatment group 2 because of $p=0.000<\alpha 0.05$. The measurement result of neutrophil count based on the Kruskal-
Willis test was $\mathrm{p}=0.001$. The above data found significant results, meaning there was a difference in the number of neutrophils in each group between the control group, treatment group 1 , and treatment group 2 because of $p=0.000<\alpha 0.05$.

Table 1: The effect of doses on the number of macrophages and neutrophils.

\begin{tabular}{lccc}
\hline Groups & Mean \pm SD & Min - Max & $\mathrm{P}$ \\
\hline Macrophage & & & \\
Control (C) & $11.36 \pm 1.43^{\mathrm{a}}$ & $9-13$ & 0.0 \\
& & & 01 \\
Treatment 1 (P1) & $13.27 \pm 1.68^{\mathrm{b}}$ & $10-15$ & \\
Treatment 2 (P2) & $16.18 \pm 1.13^{\mathrm{c}}$ & $12-19$ & \\
\hline Neutrophils & & & \\
Control (C) & $10.91 \pm 1.43^{\mathrm{a}}$ & $10-12$ & 0.0 \\
& & & 01 \\
Treatment 1 (P1) & $8.45 \pm 0.82^{\mathrm{b}}$ & $7-10$ & \\
Treatment 2 (P2) & $6.18 \pm 0.75^{\mathrm{c}}$ & $5-7$ & \\
\hline
\end{tabular}

Description: Superscript (a, b, c) show a significant difference based on the Kruskal Wallis test results ( $p$ $0.05)$

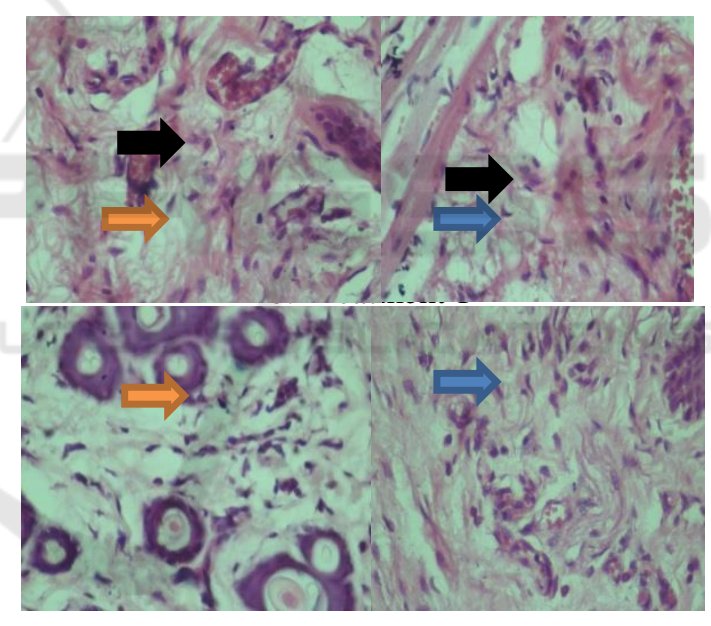

Figure 2: Macrophages and neutrophils of each group.

$\Rightarrow$ macrophages and neutrophils in the control group

$\Rightarrow \quad$ macrophages and neutrophils in treatment group 1

$\Rightarrow$ macrophages and neutrophils in treatment group 2

The anatomical histopathology results found macrophages and neutrophils. The black arrows indicate macrophages and neutrophils found in the control group. The orange arrows show macrophages and neutrophils found in treatment group 1, and the blue arrows show macrophages and neutrophils in treatment group 2 . 


\section{DISCUSSION}

This study used male rats in order for the results not to be influenced by hormones. The mice used were 8-12 weeks old because at this age facial growth and the maximum height are achieved (Lestari, 2010). This study used a type of Wistar rat because this animal has a genome sequence most similar or homologous to humans (Ridwan, 2013).

Based on Table 1 above, there were differences in the number of neutrophils and macrophages between the control group, treatment group 1 (P1), and treatment group $2(\mathrm{P} 2)$. The number of neutrophils exhibited a decrease, while the number of macrophages per group increased. This suggests chronic inflammation of the periodontal tissue after capsaicin application. In treatment group 2 (P2) high doses of capsaicin application damaged the dorsal root ganglia (DRG), because capsaicin produces neurotoxicity causing excessive calcium depolarization, whereas in treatment group $2(\mathrm{P} 2)$ low dose capsaicin application produced C-fiber which is completely reversible (Scott and Krauss, 2011).

The results of this study are in accordance with previous research which states that capsaicin Transient Potential Vanilloid-1 Receptors (TRPV1) have an effect on external damage, including traumatic stimulation, $\mathrm{pH}$ and temperature changes, and various harmful compounds. TRPV1 sensory receptors are commonly found in dental pulp, salivary glands and gingiva (Mishra and Hoon, 2010). Various diseases in the oral cavity are suspected to be due to neuropeptides as pathophysiological causes of inflammation in oral diseases (Takahashi et al., 2016). Periodontitis is one of the major inflammatory (inflammatory) diseases of the mouth characterized by inflammation of the gingiva and alveolar bone damage (Austah et al., 2016).

Hot stimuli from capsaicin cause a burning sensation caused by the interaction between the chemical compound capsaicin and neuron sensors (Mishra and Hoon, 2010). Capsaicin belonging to the vanilloid family is bound to a receptor called vanilloid receptor subtype 1 (VR 1) stimulated by heat and physical abrasion that can enter the cell membrane and cell when activated (Takahashi et al., 2016). Depolarization results from neuron stimulation of signals to the brain by binding to VR 1 , then the capsaicin molecule produces the same sensations that can cause heat and abrasion hazards, which explains why the spiciness of capsaicin is described as a spicy compound or compound that causes burning (Scott and Krauss, 2011). Channel ions from VR 1 belong to the superfamily of the Transient Receptor Potential (TRP) ion channel and is known as TRPV-1. Transient Receptor Potential has a temperature range that is acceptable for our body temperature range, so capsaicin does not cause a burning sensation like chemicals or harm body tissues; it just causes a burning sensation (Takahashi et al., 2016).

Hot stimuli induced by capsaicin may induce the release of peripheral neuropeptides. The neuropeptide-releasing effect causes vasodilation and is considered a major mediator of neurogenic inflammation (Anand and Bley, 2011). The neuropeptides contained in the fibers that supply the periodontal tissues are abundant. The production and release of these molecules are greatly increased in nocturnal, temperature, mechanical, and chemical stimulation of the dental pulp and periodontal tissues. The number of neuropeptides released by each sensory fiber is greatly increased during the inflammatory process (Austah et al., 2016).

Capsaicin produces a neurogenic inflammatory reaction mediated by axons in gingivomucous tissues (Sharma et al., 2013). Increased reaction due to capsaicin application is higher than the inflammatory effect caused by chemical irritation in the oral cavity. Wider vasoactive changes in contralateral periodontal tissue occur during different stimuli, based on the vasoactive reflex mechanism that can cause pain (Newman et al., 2015).

The TRPV reseptor was obtained in the rat's odontoblast duct. These TRPV receptors respond to thermal stimuli, extracellular stimuli or endogenous agonists. This study demonstrates the presence of thermal, pharmacological, and mechanical expression and sensitivity of TRPV receptors in the odontoblast duct (Kawashima et al., 2012). In the oral cavity, Transient Potential Vanilloid - 1 Receptors are affected by external damage, including traumatic stimulation, $\mathrm{pH}$ and temperature changes, and various harmful compounds. TRPV1 sensory receptors are commonly found in the dental pulp, salivary glands and gingiva (Mishra and Hoon, 2010).

Various diseases occurring in the oral cavity are allegedly due to the presence of neuropeptides as pathophysiologic causes of inflammation in oral diseases (Anand and Bley, 2011). Periodontitis is one of the main inflammatory (inflammatory) diseases of the mouth characterized by inflammation of the gingiva and alveolar bone destruction (Gupta, 2012). In this study, capsaicin application can 
stimulate the release of the calcitonin gene-related peptide (CGRP). CGRP is widely present in human gingival tissue, and the amount of CGRP secreted in gingival crevicular fluid (GCF) differs between subjects with and without periodontitis (Takahashi et al., 2016). Identification of CGRP in both gingival tissue and gingival sulcus fluid (GCF) is a pathological sign of periodontitis (Kawashima et al., 2012). In addition, TRPV1 is suspected to be associated with CGRP which may lead to pathophysiological interactions with inflammation of the periodontal tissues (Takahashi et al., 2016).

The inflammatory reaction occurring in topical application of capsaicin in periodontal tissues suggests a neurogenic inflammation that can trigger the expression and activation of metalloprotein-8 (MMP-8) matrix in periodontal gingival crevicular fluid (GCF) tissues. Capsaicin application in periodontal tissue can lead to significant GCF MMP8 increase and activation (Austah et al., 2016). These results suggest that irritation by capsaicin may trigger localized neurogenic inflammation with an increased proteolytic potential in periodontal tissues (Sharma et al., 2013).

In this study, capsaicin application caused inflammation in periodontal tissue characterized by the presence of neutrophils and macrophages. Inflammation of the periodontal tissue generally begins with an acute phase characterized by extravasation of proteins and plasma cells, especially neutrophils (Scott and Krauss, 2011). The functions of these factors include microbial killing and removal, necrotic tissue debridement and regeneration of damaged tissue (Walley, 2012). As inflammation progresses, there is a change from acute to chronic conditions, resulting in increased cellular defense, especially macrophages (Scott and Krauss, 2011). The presence of neutrophils is known to be a sign of periodontal damage. Found extensively within GCF and epithelium, neutrophils are considered the primary protective cell types in periodontal tissue. The neutrophil process as a defense requires adhesion, and transmigration through the blood vessel wall at the site where the vascular endothelial is activated by proinflammatory mediators (Scott and Krauss, 2011). Human junctional epithelia is never sterile, even with optimal plaque control, so neutrophils will still be stimulated to escape from the microvascular gingiva, enter into the periodontal tissue and, later, migrate first to the innerpart of periodontal tissue (Gupta, 2012). In this study, the application of capsaicin was suspected to cause neutrophils found in rat mucosa. Neutrophils undergo a glucose process to meet their energy needs while macrophages have to rely on oxidative phosphorylation. Therefore, neutrophils are like phagocytes in periodontal tissue. In essence, the stronger the inflammatory stimuli, the greater the epithelial and endothelial activation and the greater the number of recruited neutrophils (Scott and Krauss, 2011).

\section{CONCLUSION}

Capsaicin exposure has an effect on chronic inflammation in periodontal tissue (periodontitis) of white rat (Rattus novergicus).

\section{REFERENCES}

Anand, P. and Bley, K., 2011. Topical capsaicin for pain management: Therapeutic potential and mechanisms of action of the new high-concentration capsaicin 8 patch. British Journal of Anaesthesia, 107(4), pp.490502.

Austah, O.N. et al., 2016. Capsaicin-sensitive Innervation Modulates the Development of Apical Periodontitis. Journal of Endodontics, 42(10), pp.1496-1502.

Avellan, N.L. et al., 2006. Capsaicin-induced local elevations in collagenase-2 (matrix metalloproteinase8) levels in human gingival crevice fluid. Journal of Periodontal Research, 41(1), pp.33-38.

Bode, A.M. and Dong, Z., 2011. The two faces of capsaicin. Cancer Research, 71(8), pp.2809-2814.

Cunha-Cruz, J. et al., 2013. The prevalence of dentin hypersensitivity in general dental practices in the northwest United States. Journal of the American Dental Association, 144(3), pp.288-296.

Gupta, G., 2012. Gingival crevicular fluid as a periodontal diagnostic indicator--I: Host derived enzymes and tissue breakdown products. Journal of medicine and life, 5(4), pp.390-7. Available at: http://www.ncbi.nlm.nih.gov/pubmed/23346239\%5Cn http://www.pubmedcentral.nih.gov/articlerender.fcgi? artid=PMC3539845\%5Cnhttp://www.pubmedcentral.n ih.gov/articlerender.fcgi? artid=3539845\&tool=pmcent rez\&rendertype $=$ abstract.

Haggard, P. and de Boer, L., 2014. Oral somatosensory awareness. Neuroscience and Biobehavioral Reviews, 47, pp.469-484.

Kawashima, M., Imura, K. and Sato, I., 2012. Topographical organization of TRPV1immunoreactive epithelium and CGRPimmunoreactive nerve terminals in rodent tongue. European Journal of Histochemistry, 56(2), pp.129134.

Lebukan, B.J., 2013. Faktor- Faktor Penyebab Penyakit Periodontal ( Studi Kasus Masyarakat Pesisir Pantai 
Kecamatan Bacukiki Barat Kota Pare - Pare ). Jurnal Repository Unhas, pp.1-50. Available at: http://repository.unhas.ac.id/bitstream/handle/1234567 89/6445/SKRIPSI FIX.PDF?sequence=1.

Mishra, S.K. and Hoon, M.A., 2010. Ablation of TrpV1 neurons reveals their selective role in thermal pain sensation. Molecular and Cellular Neuroscience, 43(1), pp.157-163.

Newman, M.G., Takei, H.H., Klokkevold, P.R. and Carranza, F.A., 2015. Carranza's Clinical Periodontology 12th Ed,

Pashley, D.H., 2013. How can sensitive dentine become hypersensitive and can it be reversed? Journal of Dentistry, 41(SUPPL. 4).

Ridwan, E., 2013. Etika Pemanfaatan Hewan Percobaan dalam Penelitian Kesehatan. Journal Indonesian
Medical Assosiation, 63(3), pp.112-116.

Scott, D.A. and Krauss, J.L., 2011. Neutrophils in periodontal inflammation. In: Periodontal Disease. pp. 56-83.

Sharma, S.K., Vij, A.S. and Sharma, M., 2013. Mechanisms and clinical uses of capsaicin. European Journal of Pharmacology, 720(1-3), pp.55-62.

Sugiyono, 2012. Statistika Untuk Penelitian,

Takahashi, N. et al., 2016. Neuronal TRPV1 activation regulates alveolar bone resorption by suppressing osteoclastogenesis via CGRP. Scientific Reports, 6.

Walley, S., 2012. Essential microbiology for dentistry, 4th edition,

Ye, W., Feng, X.P. and Li, R., 2012. The prevalence of dentine hypersensitivity in Chinese adults. Journal of Oral Rehabilitation, 39(3), pp.182-187. 